\title{
Anesthetic management in parturients with kyphoscoliosis
}

\author{
Jay Prakash Thakur, Tara Gurung, Sangeeta Shrestha, Ujjwal Basnet, AP Pradhan \\ Department of anesthesiology, Paropakar Maternity and Women's Hospital, Thapathali, Kathmandu, Nepal.
}

Received: August 15, 2019

Accepted: December 5, 2019

\begin{abstract}
Pregnancy in kyphoscoliotic woman is uncommon, with a reported incidence of $0.072 \%$. It produces significant challenges for both anesthesiologist and obstetrician. Dealing with dual life, there is risk for both mother and fetus due to alterations in maternal physiology and change in spine anatomy. There are many case reports available with successful management both using spinal or general anesthesia. Here we present four case reports of successful management of such cases using general anesthesia or spinal anesthesia with palpatory and ultrasound guided method during a period of 6 months in Paropakar Maternity and Women's Hospital.
\end{abstract}

Keywords: Cesarean Section, kyphoscoliosis, spinal anesthesia.

Citation : Thakur JP, Gurung T, Shrestha S, Basnet U, Pradhan AP. Anesthetic management in parturients with kyphoscoliosis Nep J Obstet Gynecol. 2019;14(29):71-75. DOI: https://doi.org/10.3126/njog.v14i2.28446.

\section{INTRODUCTION}

Kyphoscoliosis is a group of spinal disorders characterized by curvature of the spine in the sagittal plane (kyphosis), lateral direction (scoliosis) as well as rotation of the spine itself. ${ }^{1}$ It may be congenital or secondary to other disorders such as polio, muscular dystrophy, cerebral palsy or idiopathic in nature. $^{1}$ Pregnancy in kyphoscoliotic lady is uncommon, with a reported incidence of $0.072 \%$. $^{2}$ Scoliosis is associated with restrictive lung disease and hypoxemia, which can lead to cardiovascular compromise. If untreated, severe scoliosis is fatal by the fifth decade of life. ${ }^{3}$ It produces significant challenges for both anesthesiologist and obstetrician. Dealing with dual life, there is risk for both mother and fetus due to alteration in maternal physiology and change in spine anatomy. There are many case reports available with successful management both using spinal and general anesthesia.

Case-1: A 27 years lady, $40 \mathrm{~kg}, 134 \mathrm{~cm}$ was referred to our hospital and admitted with diagnosis of primigravida at 42 weeks in labor with fetal distress, short stature, kyphoscoliosis, gestational hypertension and was posted for emergency Cesarean Section.

For gestational hypertension she was treated with Nifedipine. She had history of fall from tree at 7 years of age and suffered the deformity at back. There is no other significant medical and surgical history and has reasonable exercise tolerance. On examination vitals were stable, pulse 80/min, BP 140/90 mmHg, RR 16/ min, temp $98^{\circ} \mathrm{F}, \mathrm{SpO} 298 \%$. General examination and systemic examination were within normal limit. There was hump at the thoracolumbar region deviating spine towards right side. The intervertebral spaces at lumbar region were hardly palpable. With premedication and preloading, subarachnoid block was tried in sitting position using 25 gauze Quincke's spinal needle but failed. Then proceeded to general anesthesia with preoxygenation; rapid sequence induction done with inj ketamine $20 \mathrm{mg}$, inj propofol $60 \mathrm{mg}$ and inj. succinylcholine $50 \mathrm{mg}$. Cricoid pressure was applied and intubated with 6.5 $\mathrm{mm}$ endotracheal cuffed tube. Maintenance was done with oxygen, isoflurane, intermittent positive pressure

\section{CORRESPONDENCE}

Dr Jay Prakash Thakur

Department of anesthesiology

Paropakar Maternity and Women's Hospital, Thapathali,

Kathmandu, Nepal

Email: dr_jay2002@yahoo.com; Mobile: 977-9849001429 
ventilaton and vecuronium. The liquor was scanty and thick meconium stained, baby was delivered weighing $2.4 \mathrm{~kg}$ with Apgar score of $3 / 10$ and 6/10. Anesthesia reversed, and further intraoperative and postoperative period were uneventful. The baby was kept in NICU and died on 22nd day. Mother was discharged without any sequela.

Case-2: A 29 years, $122 \mathrm{~cm}, 48 \mathrm{~kg}$ lady diagnosed as $\mathrm{G}_{2} \mathrm{P}_{1} \mathrm{~L}_{0}$ at 38 weeks of gestation with cephalopelvic disproportion, moderate meconium stained liquor with kyphoscoliosis was posted for emergency Cesarean Section. Her clinical examination and investigations were within normal limit, except the spine which showed kyphoscoliotic deformity with loss of normal alignment of spines, spinous processes were hardly palpable with difficult to appreciate interspinous space as shown [Figure-1]. A 26G Quincke's needle was introduced into $\mathrm{L}_{3}-\mathrm{L}_{4}$ intervertebral space by palpatory method, clear CSF was obtained in single prick, administered $1.6 \mathrm{ml}$ of $0.5 \%$ Hyperbaric Bupivacaine, kept in supine position with support of pillow under shoulders and uterus manually displaced to left side. After 5 minutes, the sensory block was achieved up to $\mathrm{T}_{4}$ dermatome and motor block was bromage grade-4. She delivered a

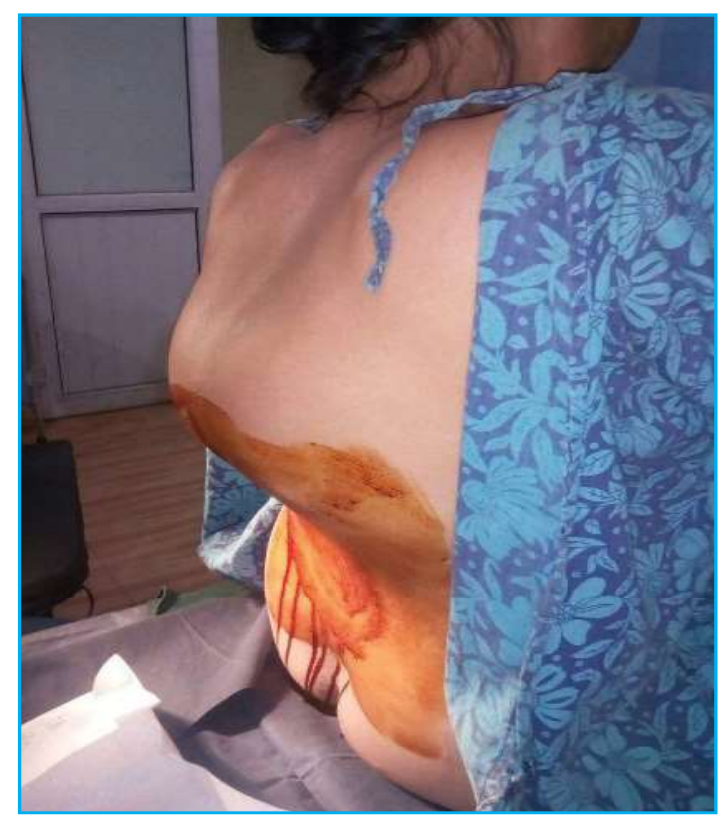

Figure-1: Patient with kyphoscoliotic deformity in sitting position

healthy baby weighing $3.5 \mathrm{~kg}$ with an Apgar score of $6 / 10$ and $8 / 10$. Further intraop and postop period were uneventful and she was discharged on $4^{\text {th }}$ postoperative day.

Case-3: A 33 years lady, $138 \mathrm{~cm}, 57 \mathrm{~kg}$ was admitted with diagnosis of $\mathrm{G}_{4} \mathrm{P}_{2+1} \mathrm{~L}_{2}$ at 40 weeks pregnancy was posted for emergency Cesarean Section for nonprogress of labour. She had history of flame burn towards right side of trunk and arm at 5 years

of age and developed contracture and kyphoscoliosis

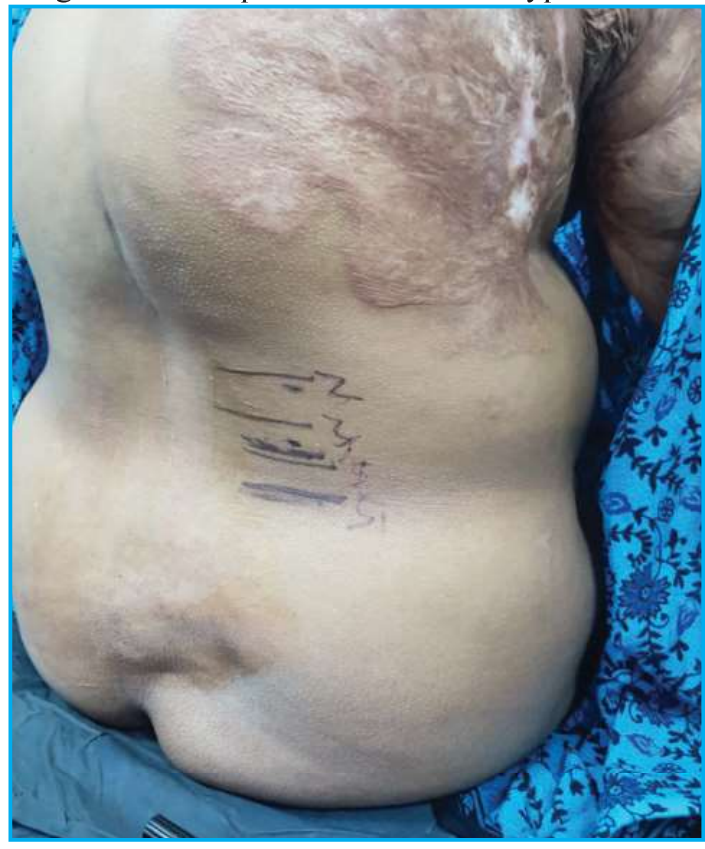

Figure-2: Back showing deformity of spine with burn contracture.

later as she grew but there was no any functional limitation. There was a hump at back. The spine was deviated towards right side and intervertebral spaces were palpable at lumbar region. Subarachnoid block performed in sitting position with the aid of ultrasonography to identify L3-L4 intervertebral space using 27 gauze whitacre spinal needle with inj. Bupivacaine $0.5 \%$ heavy $1.6 \mathrm{ml}$. The free flow of CSF was obtained at single prick [Figure-2]. The block height was upto at T4 sensory level. Healthy baby was delivered. The intraoperative and postoperative periods were uneventful. The mother and baby were discharged on $5^{\text {th }}$ postoperative day.

Case-4: A 32 years lady, $148 \mathrm{~cm}, 58 \mathrm{~kg}$ with diagnosis $\mathrm{G}_{5} \mathrm{P}_{4} \mathrm{~L}_{1}$ at 40 weeks pregnancy with bad obstetric history was posted for elective Cesarean Section. She had history of fall from roof at 10 years of age and she gradually developed kyphoscoliosis. She was kept in sitting position, the intervertebral space were very 
difficult to palpate and there was right sided shift of spine. With the aid of Ultrasonography intervertebral space was identified at L3-L4 level and subarachnoid block was given with 27 gauze whitacre spinal needle [Figure-4]. With inj. Bupivacaine $0.5 \%$ heavy $1.4 \mathrm{ml}$

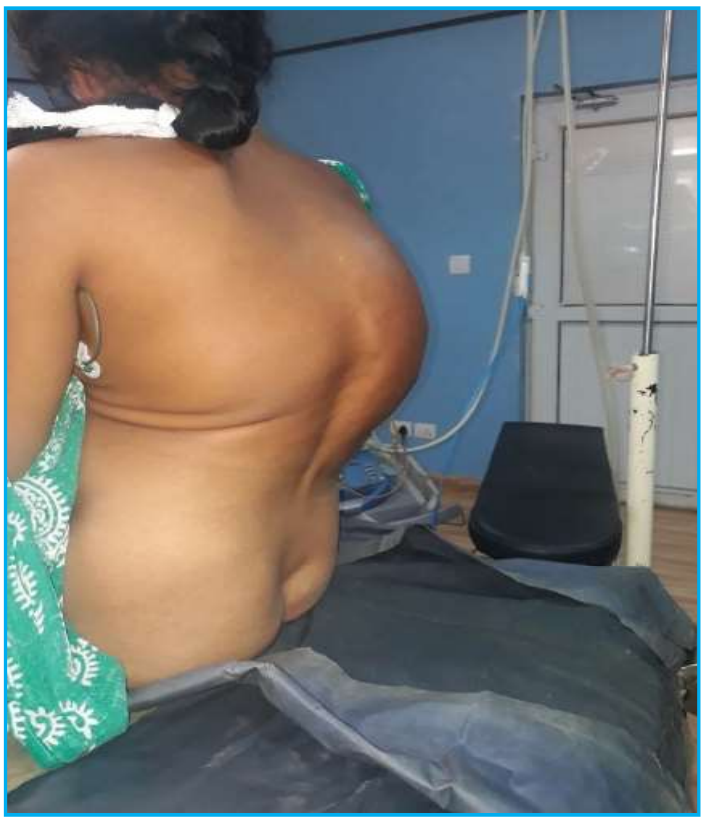

Figure-3: Patient with kyphoscoliotic deformity.

+ inj Fentanyl $0.4 \mathrm{ml}(20 \mathrm{mcg})$ the sensory block was

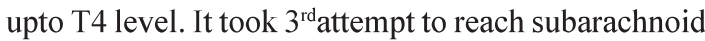
space, every time bone was encountered, finally succeeded after tilting needle on towards right side towards spinous deviation. Further intraoperative

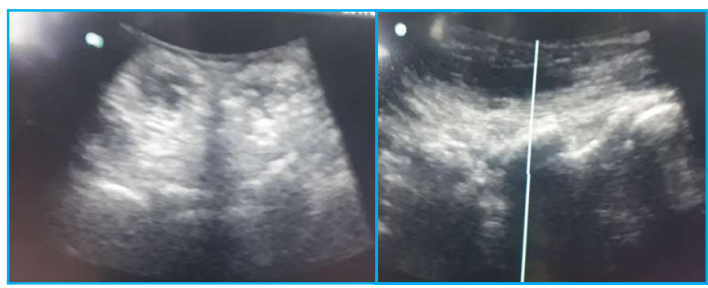

Figure-4: Ultrasound picture of lumber spines

and postoperative period were uneventful. She was discharged on $5^{\text {th }}$ postoperative day.

\section{DISCUSSION}

At present, most Cesarean Sections are performed with neuraxial techniques under subarachnoid block. Parturients who undergo general anesthesia for cesarean delivery are at higher risk for mortality due to pulmonary aspiration of gastric contents, failed intubation of the trachea or inadequate ventilation compared with those under neuraxial blockade, particularly in emergent situations. ${ }^{4}$

Kyphoscoliosis produces a significant challenge to the anesthesiologist. Pregnancy may exacerbate the severity of spinal curvature in women with kyphoscoliosis. ${ }^{3}$ Cobb's angle best determines the severity of scoliosis which is defined as the angle between the perpendiculars of the lines drawn parallel to the upper border of the highest and lower border of the lowest vertebrae [Figure-5]. ${ }^{5}$

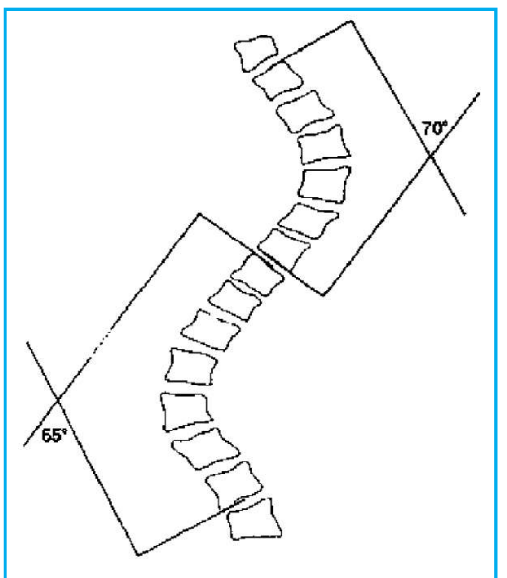

Figure-5: Cobb's method: measurement of severity ${ }^{6}$

The increasing severity of scoliosis produces clinical manifestation from asymptomatic up to respiratory failure [Table-1]. ${ }^{?}$

Table-1: severity of scoliosis and clinical correlation. ${ }^{7}$

\begin{tabular}{|l|l|}
\hline $\begin{array}{l}\text { Cobb's angle } \\
\text { (in degrees) }\end{array}$ & Clinical manifestations \\
\hline$<10$ & No symptoms \\
\hline$>25$ & Increase in pulmonary artery pressure \\
\hline$>40$ & Consider surgical intervention \\
\hline$>70$ & Significant decrease in lung volume \\
\hline$>100$ & Dyspnea on exertion \\
\hline$>120$ & $\begin{array}{l}\text { Alveolar hypoventilation, chronic } \\
\text { respiratory failure }\end{array}$ \\
\hline
\end{tabular}

Weinstein and colleagues reported that Cobb's angle above 60 degrees results in progressive pulmonary and cardiac failure. ${ }^{8}$ Harrison et al reported that pulmonary impairments are most notable at kyphosis angles $>55$ degrees. ${ }^{9}$ 
Scoliosis involving upper thoracic or cervical spine can cause difficult airway. ${ }^{6}$ In severe cases, difficulty during laryngoscopy and intubation may encounter due to displacement and rotation of the trachea and main stem bronchi. ${ }^{3}$ For each case we encountered, although the airway doesn't seem difficult but still we had prepared difficult airway equipment cart in case if it is required.

Kyphoscoliotic patients suffer from restrictive lung disease which involves decreased tidal volume, functional residual capacity, vital capacity and increased respiratory rate. ${ }^{6}$ The Cobb's angle, the cephalic location of the curvature and the number of vertebrae involved determines severity of pulmonary impairment. ${ }^{3}$ In normal parturient lady, minute ventilation volume increases by $40-50 \%$ due to increase in tidal volume with relatively unchanged respiratory rate mainly achieved by alterations in the thoracoabdominal architecture. These adaptations are not possible in kyphoscoliotic patients. ${ }^{10}$ As the disease progresses gas exchange is affected by alveolar hypoventilation, an increased dead space, ventilationperfusion mismatch, and an increased alveolararterial gradient. Prolonged periods of hypoxemia result in pulmonary hypertension, hypercapnia and eventual respiratory failure. ${ }^{6}$ These cardiopulmonary changes result in increased maternal morbidity as well as requirement for the assisted ventilation. ${ }^{11}$ However, successful pregnancy without ventilator support has also been reported. ${ }^{12}$ Care should be taken to avoid hypoxia, hypercapnia, acidosis, and anesthetic gases such as nitrous oxide as they increase the pulmonary vascular resistance. ${ }^{3}$ Respiratory system compliance decreases because of the distortion of the thoracic cage, thus increasing the work of breathing even when the lungs themselves are healthy. ${ }^{13} \mathrm{All}$ our parturients had acquired kyphoscoliosis and no any respiratory symptoms, so further evaluation of respiratory reserve was not done; otherwise Pulmonary Function Test and Arterial Blood Gas analysis is advisable before surgery.

Scoliosis is associated with changes in the mediastinum leading to restrictive pericarditis. Pulmonary hypertension occur secondary to the chronic respiratory insufficiency. So, cardiac reserves are limited and may not be able to withstand the increased hemodynamic demand. ${ }^{6}$ Cardiac output increases during pregnancy more during the last trimester which further increases the pulmonary artery pressure leading to intolerable load on right ventricle and precipitating the right heart failure. Fixed pulmonary hypertension, unresponsive to oxygen therapy carries a grave prognosis and is an indication for termination of pregnancy. Death during peripartum period is common in parturient with pulmonary hypertension. ${ }^{14}$ All our parturients have good functional capacity suggesting good cardiopulmonary reserve.

Cesarean Section in a severely kyphoscoliotic patient also presents with unique challenges to obstetrician. Supine positioning of such patient is difficult. Baby should be delivered by incision on the most easily accessible part of the uterus. Postpartum hemorrhage should be anticipated as mostly present with obstructed labour and the obstetrician should be prepared for the same. ${ }^{15}$

Neuraxial blockade in patient with kyphoscoliosis has complications such as unpredictable block,risk of high spinal anesthesia, multiple attempts and failure rates. Severe kyphoscoliosis can be associated with decreased volume of cerebrospinal fluid and even lower doses of local anaesthetics may achieve higher than expected level of block with more incidence of hypotension..$^{10}$ Adjusting the doses is desirable. There are reports that in patients with severe curves hyperbaric solution may pool in the dependent portion of the spine and results in inadequate block. ${ }^{16}$ We were unable to find CSF in $1^{\text {st }}$ case but were lucky in other cases to get CSF and also the level of blocks were adequate. Neuraxial ultrasound can be used to accurately identify lumbar intervertebral levels, precise measurement of depth of epidural space, improve the efficacy and safety of central neuraxial block and decrease the number of needle redirections and skin punctures. ${ }^{17}$ However for use of ultrasound guided neuraxial blocks, it requires technical expertise. In our $3^{\text {rd }}$ case we successfully used neuraxial ultrasound, but in $4^{\text {th }}$ case despite its use we could get CSF only in $3^{\text {rd }}$ attempt.

General anesthesia is indicated in scoliosis because of maternal preference, when there is maternal cardiopulmonary disease and when there is difficulty in performing regional block. ${ }^{3}$ But there can be hazardous increase in pulmonary artery pressure leading to right heart failure. Positive pressure ventilation decreases venous return and along with negative ionotropic effect of anaesthetic agents 
can lead to severe decrease in blood pressure. Coughing and bucking at the end of the surgery may transiently but significantly decreases functional residual capacity, resulting in further ventilation perfusion mismatch and hypoxemia. Postoperatively after general anaesthesia, elements of laryngeal incompetence and impaired swallowing further decrease the airway defense mechanisms. All these factors together can lead to delay in extubation and need for postoperative ventilation. ${ }^{10}$ In our $1^{\text {st }}$ case as we failed to perform spinal anesthesia and was converted to general anesthesia, it was successfully performed as she had good cardiopulmonary reserve.

\section{CONCLUSIONS}

To conclude, this report supports the feasibility of both spinal anesthesia and general anesthesia in parturients with kyphoscoliosis as per the situation demands but preference should be given to spinal anesthesia especially with the aid of neuraxial ultrasound. Cardiopulmonary reserve and ultrasound guidance should be reassuring for the successful anesthesia in kyphoscoliosis.

\section{REFERENCE}

1. Tzelepis GE, McCool FD. Nonmuscular deseases of the Chest Wall. In: Fishman AP, Elias JA, Senior RM, Fishman JA, Pack AI, Grippi MA, editors. Fishman's Pulmonary Diseases and Disorders. 4th ed. United States of America: Mc Graw Hill 2008. P. 1617-33

2. Chopra S, Adhikari K, Agarwal N, Suri V, Sikka P Kyphoscoliosis complicating pregnancy: maternal and neonatal outcome. Archiv Gynecol Obstet. 2011;284(2):2957. Epub 2010/08/17

3. Vagyannavar R, Devi R. A case report of emergency Cesarean Section in parturient with kyphoscoliosis. Med J DY Patil Univ. 2017;10:300-2.

4. Flood P, Rollins MD. Anesthesia for Obstetrics. IIn: Miller RD, Cohen NH, Eriksson LI, Fleisher LA, WienerKronish JP, Young WL, editors. Miller's Anesthesia. 8th ed. Philadelphia Saunders, Elsevier; 2015. p. 2328-58.

5. Gupta S, Singariya G. Kyphoscoliosis and pregnancy- a case report. Indian J Anaesth. 2004;48(3):215-220

6. Kulkarni AH, Ambareesha M. Scoliosis and anesthetic considerations. Indian J Anaesth. 2007;51:486-95.

7. Koumbourlis AC. Review: Scoliosis and the respiratory system. Paed Resp Rev. 2006;7:152-60

8. Weinstein SL, Zavala DC, Ponseti IV. Idiopathic scoliosis long term follow up and prognosis in untreated patients. J Bone Joint Am. 1981:63:702-12
9. Harrison RA, Siminoski K, Vethanayagam D. Osteoporosisrelated kyphoscoliosis and impairement in pulmonary function a systematic review. J Bone Miner RES. 2007;22(3):447-57.

10. 10. Bansal N, Gupta S. Anaesthetic management of a parturient with severe kyphoscoliosis. Kathmandu Univ Med J. 2008;6(23):379-82. Epub 2008/07/01.

11. Kahler CM, Hogl B, Hobeler R. Management of respiratory deterioration in a pregnant patient with severe kyphoscoliosis by non invasive positive pressure ventilation. Wien Klin Wochenschr. 2002;114(19-20):874-7

12. Yim R, Kirschner K, Murphy E. Successful pregnancy in a patient with spinal muscular atrophy and severe kyphoscoliosis. Am J Phys Med Rehabil. 2003;82(2):222-5.

13. Cooper DM, Rojas JV, Mellins R. Respiratory mechanics in adolescents with idiopathic scoliosis. Am Rev Respir Dis $1984 ; 130: 16-22$

14. Gummerus M, Laasonen $H$. Eisenmenger complex and pregnancy. Ann Chir Gynaecol. 1981;70:339

15. Chhetry M, Banerjee B, Subedi S. Challenges in the Caesarean Section of a Severely Kyphotic Parturient. Case Rep Obstet Gynecol. 2016.

16. Moran DH, Johnson MD. Continuous spinal anaesthesia with combined hyperbaric and isobaric bupivacaine in a patient with scoliosis. Anesth Analg. 1990;70:445

17. SM Ghosh, C Madjdpour, KJ Chin. Ultrasound guided lumbar central neuraxial block. British J Anesth. 2015;16(7):213-220. 